\title{
Evaluating the Effectiveness of Information Presentation in a Full End-To-End Dialogue System
}

\author{
Taghi Paksima \\ Kallirroi Georgila \\ Johanna D. Moore \\ Enterprise Search Group \\ Microsoft \\ Institute for Creative Technologies \\ University of Southern California \\ School of Informatics \\ D-81669 Munich, Germany Marina del Rey, CA 90292, USA Edinburgh, EH8 9AB, UK \\ taghi.paksima@microsoft.com kgeorgila@ict.usc.edu_j.moore@ed.ac.uk
}

\begin{abstract}
Recent work on information presentation in dialogue systems combines user modelling (UM) and stepwise refinement through clustering and summarisation (SR) in the UMSR approach. An evaluation in which participants rated dialogue transcripts showed that UMSR presents complex trade-offs understandably, provides users with a good overview of their options, and increases users' confidence that all relevant options have been presented (Demberg and Moore, 2006). In this paper, we evaluate the effectiveness of the UMSR approach in a more realistic setting, by incorporating this information presentation technique into a full endto-end dialogue system in the city information domain, and comparing it with the traditional approach of presenting information sequentially. Our results suggest that despite complications associated with a real dialogue system setting, the UMSR model retains its advantages.
\end{abstract}

\section{Introduction}

Spoken dialogue systems (SDS) that help users find a desired option (e.g., flight, restaurant, movie) from the set of options satisfying their constraints typically present options sequentially, ordered along a default dimension (e.g., by price or departure time). An example is shown in Fig. 1.

The user can then navigate through the options and refine them by offering new constraints until a suitable option has been found. However, when the number of available options is large, this process can be painstaking, leading to long dialogues
There are six restaurant options matching your query.

Number 1: Voujon offers a bright, airy and contemporary dining area, with simple floral displays and leather seating. It serves Indian cuisine. It is located in the city centre. The average price is $£ 24$ per person.

Number 2: Saffrani's decor is modern, the dining room wee, though the menu is enormous, and the atmosphere charming. It offers new Indian dishes never before seen in Edinburgh. It serves Indian, seafood cuisine. It is located in the city centre. The average price is $£ 28$ per person.

Number 3: Britannia Spice ...

Figure 1: Example of sequential information presentation in the city information domain (modified version of the TownInfo system (Lemon et al., 2006)).

and reduced user satisfaction. Thus a major challenge in the development of SDS is to improve information presentation algorithms. This is important for several reasons: (1) to avoid overburdening the user's memory by presenting too many options; (2) to ensure that the user is given an overview of the available option space so that the optimal option can be found; and (3) to minimise the number of dialogue turns (hence dialogue duration) required for the user to find an acceptable option. As Walker et al. (2001) showed, failing to meet this third goal may reduce overall user satisfaction.

Recently several approaches have been proposed to overcome the shortcomings of the sequential enumeration strategy (Polifroni et al., 2003; Chung, 2004; Demberg and Moore, 2006; Polifroni and Walker, 2008). Because of the complexity of building a complete end-to-end SDS, these approaches have been evaluated using an "overhearer" methodology in which dialogues are either hand-crafted or simulated and then presented to subjects, either as textual transcripts 
(Demberg and Moore, 2006; Polifroni and Walker, 2008) or audio recordings (Walker et al., 2004), for rating. The general consensus from these studies is that users significantly prefer approaches that take their preferences into account. However, because users were not interacting with these SDS, the evaluation criteria were limited to users' perceptions (e.g., informativeness, good overview of options, confidence in choice, etc.), and metrics such as effectiveness (i.e., actual or perceived task completion) and efficiency (i.e., length of dialogue) could not be assessed. To address this issue, Winterboer and Moore (2007) carried out a Wizard-of-Oz (WOz) study in which users participated in dialogues controlled by two different information presentation algorithms. They found that not only did users prefer presentations based on a user model, dialogues employing the "usermodel based summarise and refine" (UMSR) approach led to greater task success and dialogue efficiency.

In this paper, we take this one step further, and evaluate the effectiveness of the UMSR approach in a more realistic setting, incorporating this content selection and presentation strategy into a full end-to-end dialogue system, and comparing it to the traditional sequential enumeration approach. Our results suggest that despite complications associated with a real dialogue system setting, the UMSR model retains its advantages. Our results also verify the hypothesis that the UMSR model presents complex trade-offs in a concise, yet understandable way. Furthermore, as in the $\mathrm{WOz}$ study, the UMSR approach leads to a significant reduction in the number of dialogue turns.

The structure of the paper is as follows: In Sec. 2, we discuss related work. In Sec. 3 we present the full end-to-end SDS used for comparison between the standard sequential enumeration approach and the UMSR approach. In Sec. 4 we describe how we implemented the UMSR approach. Then in Sec. 5 we provide an example. In Sec. 6 we describe our experimental design and in Sec. 7 our results. Finally in Sec. 8, we present our conclusions.

\section{Previous Approaches}

As noted above, a number of approaches to information presentation in SDS have recently been proposed. The user-model based (UM) approach employs a model of the users preferences and decision theory techniques to identify and present a small number of options that best match the user's preferences (Carenini and Moore, 2001; Walker et al., 2004; Moore et al., 2004). Fig. 2 shows a sample presentation generated using the UM approach for a student user who cares most about price and flying direct.

There's a direct flight on BMI with a good price. It arrives at four ten p.m. and costs a hundred and twelve pounds. The cheapest flight is on Ryanair. It arrives at twelve forty-five p.m. and costs just fifty pounds, but it requires a connection in Dublin.

Figure 2: Example of information presentation based on the UM approach, from (Moore et al., 2004).

Although the UM approach enables a concise presentation of a small number of options, highlighting the ways in which these options satisfy user preferences, it does not scale up to presenting a large number of options. When there are hundreds of potentially relevant options to consider (e.g., all Italian restaurants in New York City), there may be a large number of options that fit the user model, making it difficult to narrow down the number of options. In addition, users may not be able to provide constraints until they are presented with more details concerning the option space. Moreover, the system does not provide an overview of the available options, which may lead to the user's actual or perceived missing out on possibly better alternatives.

An alternative approach, which we call the summarise and refine approach (SR) (Polifroni et al., 2003; Chung, 2004) structures large datasets for summarisation and successive refinement. It supports the user in narrowing in on a suitable option by grouping the options in the database into clusters of options with similar features. The system can then summarise the common attributes of the options and thus suggest further refinement constraints to the user. In this content structuring approach, the prompts presented to the user and the order in which they appear, are determined at runtime based on an algorithm that computes the most useful set of attributes, as dictated by the set of options that satisfy the user query. For large datasets, attributes that partition the data into the minimal number of clusters are chosen, so that a concise summary can be presented to the user to refine (Fig. 3).

In addition to its many advantages, the SR approach also has several limitations. First, depending on the size and complexity of the domain, many turns may be required during the refinement 
M: What restaurants do you have?

S: I have found 983 restaurants. Most of them are located in Boston and Cambridge. There are 32 choices for cuisine. I also have information about price range.

M: Okay tell me about the ones in Boston.

S: I have found 401 restaurants in Boston. There are 29 choices for cuisine.

M: Do you have any that serve seafood?

S: I have found 19 seafood restaurants. They are predominantly in Back Bay, the North End, South Boston and the South End.

Figure 3: Sample dialogue between simulator (M) and SR system (S), from (Polifroni et al., 2003).

process. Because the user's preferences are not taken into account, the clusters may contain many irrelevant entities, which must be filtered out successively with each refinement step. Second, if there is no optimal solution, exploration of tradeoffs between options can be difficult. Finally, the chosen clusters may be based on attributes that are irrelevant for the specific user.

In an attempt to combine the benefits of the UM and SR approaches, Demberg \& Moore (2006) devised the user-model based summarise and refine (UMSR) approach to information presentation. This approach first clusters the values of each attribute in order to group them so that the options can be summarised more easily later, and labels like "cheap", "moderate", "expensive" can be assigned to values of continuous categories such as "price". The system then structures options into an option tree based on the ranking of attributes in the user model, the options returned from the database, and the attribute-value clustering. The resulting option tree determines how different options relate to one another, and which ones are most attractive for the user. After the tree structure is built, it is pruned to decide which options are compelling to the user according to the user model. This allows the system to save time by omitting options that are not of any potential interest to the user. Once pruning is complete, each branch of the tree describes a possible refinement path, and thus can be used to direct dialogue flow. Trade-offs between alternative options are presented explicitly in order to provide the user with a better overview of the option space. In addition, to give users confidence that they are being presented with all of the relevant options, a brief account of all the remaining (irrelevant) options is also provided. For a more detailed discussion of the UMSR approach, see (Demberg and Moore,
2006). In Sec. 4 we describe how we employed the UMSR approach in our system.

\section{The TownInfo System}

The TownInfo SDS was developed as part of the EC project TALK (Lemon et al., 2006). Users can search for hotels, bars and restaurants in an artificial town. The system supports two dialogue strategies, one hand-crafted and another learnt using Reinforcement Learning (Henderson et al., 2008). For the current experiment we used the hand-crafted strategy. Natural language understanding is performed using a keyword-based parser and natural language generation is based on templates. The information presentation is sequential. An example is given in Fig. 1, taken from the modified version of TownInfo for the current experiment. Although the original TownInfo system supported speech input and speech output, here we use text input/output to make sure that our results are not influenced by poor recognition accuracy or intelligibility due to poor speech synthesis. Of course, as we mention in Sec. 8, the next step would be to perform an experiment with speech input/output.

For our current experiment we focussed on restaurant recommendations and the TownInfo database had to be extended to include a much wider range of options to provide more realistic information presentation scenarios. The database used in our experiments contains a total of 80 restaurants in Edinburgh, UK.

\section{The UMSR Algorithm}

This section briefly describes our implementation of the UMSR algorithm; for more details see (Demberg and Moore, 2006). Sec. 5 provides an example for clarity.

\subsection{The User Model}

The user model contains the user's ranking and preferred values for the relevant attributes in the restaurant domain: price, distance, star rating, service rating, and cuisine type. Table 1 shows a sample user model. The Rank field indicates the relative importance of the attributes for the user, with 1 being most important. The Value field indicates the user's preferred value for each attribute. ${ }^{1}$

\footnotetext{
${ }^{1}$ If two attributes in a user model have identical ranks, the order of the preferences is used to decide which has a higher priority.
} 


\begin{tabular}{|cccc|}
\hline UserID & Attribute & Value & Rank \\
\hline 1 & Price & Cheap & 1.00 \\
1 & Distance & Near & 2.00 \\
1 & Star & High & 3.00 \\
1 & Cuisine & Indian & 4.00 \\
1 & Service & Don't Care & 5.00 \\
\hline
\end{tabular}

Table 1: Sample user model for a student.

According to Elzer et al. (1994), some preferences are enough to reject options outright (and therefore are more like goals) whereas others are more purely like preferences (to be weighed and ranked). Here we do not make such a distinction.

\subsection{Adapting to Changes to the User Model}

In the original design, the user model was created at the outset and not modified during the dialogue. However, during initial piloting of the system, we found that this design did not support "situational preferences". For example, consider the user model for the student in Table 1. This user normally prefers to have Indian food if she has the option to (a "dispositional preference"). If, however, in the current situation she is entertaining a friend from out of town who wishes to try Scottish food, the user may decide to explore options for Scottish cuisine (a "situational preference"). Here, the user changes her original query for the situation, thus redefining her preferences. When this occurs, we must perform a new database query and rebuild the option tree. To take these dynamic changes into account during the course of the dialogue, at each dialogue turn the user query is compared against the user model, and if any difference is noted, the user model is updated to reflect the current preferences, the tree is rebuilt using the new user model, and the dialogue continues with a summary of the available options based on this new tree.

Note that for individual models, i.e. user models that are designed for individual people and not for classes of users (student or business person), some queries could justify situational changes and some could indicate permanent (or at least less temporary) changes to the user model (e.g., "Are there any nicer restaurants? I got a new job"). In our experiment we use only class models and we do not allow permanent changes to the user model.

\subsection{The Clustering Algorithm}

Following (Polifroni et al., 2003) and (Demberg and Moore, 2006), we used agglomerative groupaverage clustering to automatically group values for each attribute. The algorithm begins by assigning each unique attribute value to its own bin, and successively merging bins whose means are most similar until a stopping criterion (a target of no more than three clusters, in our implementation) is met. The bins are then assigned predefined labels, e.g., "cheap", "moderately priced" and "expensive" for price. Clustering attribute values with this algorithm allows for database-dependent labelling. Therefore, a restaurant with a price of $£ 35$ might be considered as expensive for Edinburgh, but inexpensive for London.

\subsection{Building the Option Tree}

The tree building algorithm is recursive. It begins at the root node, which contains all entities in the retrieved dataset, and builds up the tree level by level based on the ranking of attributes in the user model. At each node of the tree, it retrieves the next attribute preference from the user model and then invokes the clustering algorithm for this attribute's values. Once the current dataset has been clustered, the algorithm then adds the resultant clusters as the children of the current node. After each cluster is added, the algorithm is invoked recursively on the newly created children of the current node.

As the tree is being constructed, the algorithm arranges the nodes in the tree such that the children of each node are ordered from left to right in decreasing order of desirability. For example, if the particular user prefers restaurants that are far from the city centre, the clusters based on distance would be ordered such that "far" is the leftmost child and "near" is the rightmost child. Fig. 5 depicts an option tree structure for the user model of Table 1, in the context of the example of Sec. 5. The numbers in the nodes indicate how many options are represented by the node.

Given an option tree ordered in this way, to find the best available options, the system traverses the tree in a depth-first fashion starting from the root and selecting the leftmost branch at each node.

\subsection{Pruning the Option Tree}

The goal of the UMSR algorithm is to present an overview of the available options, that are most relevant to the user's preferences, concisely and understandably. To determine the relevance of options, we use the notion of "dominance" defined in Demberg \& Moore (2006). Dominant options are those for which there is no other option in the dataset that is better on all attributes. A domi- 
nated option is in all respects equal to or worse than some other option in the relevant subset of the database; it should not be of interest for any rational user.

The pruning algorithm follows Demberg \& Moore (2006), and thus we summarise it only briefly here. The algorithm operates directly on the ordered option tree, using the tree structure so that it can efficiently determine dominance relations without having to compare each pair of options. The algorithm traverses the tree in depthfirst order, generating constraints during this process. These constraints encode the properties that other options would need to satisfy in order not to be dominated by the options which have already been deemed to be dominant. A node must fulfil the constraints that apply to it, otherwise it is pruned from the tree. If an option (or a cluster of options) satisfies a constraint, the property that satisfied the constraint is marked as the options' justification. If some, but not all, of the constraints can be satisfied by an option, the constraints are propagated to the other nodes (see Fig. 5).

\subsection{Natural Language Generation}

Once a pruned option tree has been constructed, the system can generate a presentation to the user. The natural language generation (NLG) algorithm includes three steps described below.

\subsubsection{Identifying Trade-offs}

To identify the trade-offs, the algorithm traverses the tree looking for constraints that were generated during the pruning process. For each node that generated a constraint, the algorithm finds the best sibling, which satisfies the constraint. It does this by first checking the siblings of the current node, and if none satisfy the constraint, it moves up the tree and recursively traverses siblings of the ancestor node. Once a tradeoff node is found, it is recorded in the option tree at that point, and the algorithm then searches upward in the tree to find the lowest common parent of the trade-off nodes. This is the "Differentiating Parent" for the trade-off; the attribute that determines the children of this node indicates the tradeoff the user has to make. In the example shown in Fig. 5, the attribute that the user must tradeoff is distance. The system then generates a natural language description of the trade-off using templates, e.g., "For a restaurant that serves Indian cuisine you would have to go farther from the city centre". If a constraint cannot be satisfied, the sys- tem generates a sentence describing this situation, e.g., "However, there are no inexpensive restaurants that match your preferences".

\subsubsection{Summarisation}

The summarisation process aims to present the user with an understandable and concise overview of the available options, so that she can make informed decisions on how to proceed with her query. In a SDS, it is important not to present too much information in a single turn in order to keep the memory load on the user manageable. Thus, in our implementation, the system provides a summary of the first three attributes (corresponding to the first three levels of the tree), e.g., "I found 12 restaurants which are inexpensive. Only 7 of them are near the city centre, out of which only 4 have a high star rating". If there are any trade-offs over the first three attributes, they are also mentioned to the user, using the algorithm described above.

\subsubsection{Turn Generation}

The turn generation algorithm first determines whether the current user's query is consistent with the preferences defined in the user model. Since the option tree, and consequently the generation so far, have primarily been based on the user model, if the algorithm notices an inconsistency it will give priority to the most recent user query as a situational adjustment (see Sec. 4.2), and then update the user model to reflect the adjustment. If the user model is modified, the algorithm rebuilds the option tree, and generates the next turn using the new user model and the most recent user query.

To ensure the user that the system has understood her correctly, and to increase her confidence, the algorithm includes a summary of all attribute values that are common to the cluster in the option tree that is being used to generate the summary. For example, for the second turn in the dialogue of Fig. 4, although the user's most recent query was about cuisine, the system includes a summary of all the attributes already mentioned to the user: "All of them are cheap, are near the city centre, and have a high star rating".

\section{Example}

To clarify things further, here we present an example. A dialogue between the UMSR system and a student (user model of Table 1) is depicted in Fig. 4.

Fig. 5 depicts the tree structure for the student user of our example. The user begins by 
U: Tell me about the restaurants you have.

S: I found 12 restaurants which are inexpensive. Only 7 of them are near the city centre, out of which only 4 have a high star rating. I also have information about cuisine and service quality.

U: Tell me about the cuisine.

S: There is only 1 restaurant that serves Scottish food, and 3 restaurants that serve French food. They all provide good service quality. All of them are cheap are near the city centre, and have a high star rating. For a restaurant that serves Indian cuisine you would have to go farther from the city centre.

U: Okay, tell me about the Scottish one.

S: The Jam House's menu includes Scottish dishes sparked with international flavour, with a decent choice of vegetarian dishes. It is located in the centre. The average price is $£ 22$ per person.

U: What about Indian restaurants?

S: I found 2 restaurants that serve Indian food. Both of them are cheap, within average distance from the city centre, and have a high star rating. Only one of them provides good service quality.

$\mathrm{U}$ : Okay, tell me about this one.

S: The Raj on the Shore's theme extends from the soldier figurines behind the exotic blue bar to the predominantly Bangladeshi, Indian menu. It serves Indian and Bangladeshi cuisine. It is located in the north. The average price is $£ 20$ per person.

Figure 4: Example dialogue with our UMSR approach (student user of Table 1).

asking generally for a restaurant recommendation, and the system provides a summary based on the three top-ranking attributes for this user: price, distance and star rating, which correspond to the first three levels of the option tree. Next the user asks about cuisine type, which generates a trade-off since there are no Indian restaurants (user's preference) that are cheap, near the city centre, and of high star rating. The user then asks about the Scottish option, before switching back to her preferred cuisine type (Indian). Because Indian cuisine was in the user's initial preference model, a constraint of cuis ine=Indian was generated when traversing the leftmost branch of the tree, and this justified not pruning the unshaded nodes in the right subtree of Fig. 5, in order to generate the trade-off. However, if the user had asked about expensive restaurants, then a new database query would have been made and a new option tree would have been built. A more complex example is given in the Appendix.

\section{Experimental Design}

In total 18 subjects interacted with our two systems. Each participant interacted three times with the modified TownInfo system, and another three times with the system that supported our implementation of the UMSR model (108 dialogues in

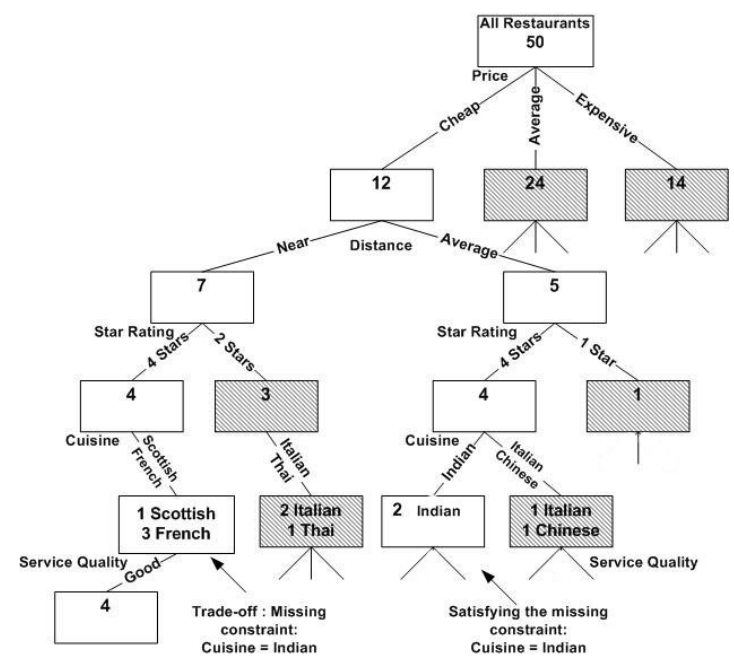

Figure 5: A sample option tree structure for the student user of Table 1. Pruned nodes are shown as shaded.

total). The order of the dialogues was randomised among the subjects. Each experiment took between 40 and 50 minutes on average.

For each task, subjects were provided with the user profile and the actual scenario for the specific task in hand. The tasks were carefully constructed so that half of them could be solved without making any trade-offs and the other half required a trade-off to be made. At the end of each task the subjects had to fill out a questionnaire with 10 questions on a 7-point Likert scale. They were also asked if they had been able to accomplish the given task (perceived task completion), i.e., to find a suitable restaurant for the scenario and user profile in hand. Finally, after each task they had to provide the name(s) of the restaurants they chose for the task. The name(s) stated for this task were then used to compare perceived task completion with actual task completion. At the end of each task with the UMSR system, the profiles were reset to the default attribute values and ranks.

Both systems had identical software configurations, i.e., they only differed in the information presentation component. Yet another important feature was that the UMSR based model did not accept multiple attributes in a single query. So for instance the user could not ask "I am looking for a moderately priced restaurant near the city centre that serves Italian food". This seemed to be a major shortcoming of the UMSR based system compared to the TownInfo system with sequential information presentation. However, as we will see in the following, even with this shortcom- 


\begin{tabular}{|l|ccccc|}
\hline System & $\mathbf{U}$ & $\mathbf{C C}$ & $\mathbf{C F}$ & $\mathbf{A}$ & $\mathbf{E}$ \\
\hline UMSR-all & 5.04 & 4.65 & 3.22 & 3.66 & 4.69 \\
TownInfo-all & 4.87 & 4.04 & 2.93 & 3.20 & 3.59 \\
UMSR-with TO & 4.74 & 4.59 & 2.67 & 3.26 & 4.15 \\
TownInfo-with TO & 4.59 & 3.41 & 2.74 & 2.33 & 2.70 \\
UMSR-no TO & 5.33 & 4.70 & 3.78 & 4.08 & 5.22 \\
TownInfo-no TO & 5.15 & 4.67 & 3.11 & 4.07 & 4.48 \\
\hline
\end{tabular}

Table 2: Average scores of the questionnaires for all dialogues, dialogues with tradeoffs (with TO) and dialogues without trade-offs (no TO) ( $\mathrm{U}=$ understandability, $\mathrm{CC}=$ conciseness, $\mathrm{CF}=$ confidence, $\mathrm{A}=$ accessibility, $\mathrm{E}=$ efficiency).

ing the UMSR approach retained its advantages and proved more successful than the traditional sequential enumeration approach.

\section{Results}

The perceived task completion (PTC) for the UMSR system and the TownInfo system was $90.74 \%$ and $85.19 \%$ respectively, and the actual task completion (ATC) $74.07 \%$ and $62.96 \%$. Thus the UMSR approach led to a relatively better user confidence in having achieved the task.

The average number of turns was 9.24 for UMSR compared to 17.78 for TownInfo, which denotes a significant reduction in the number of dialogue turns required to accomplish a given task. This reduction becomes even more prominent when there is a trade-off involved. With such dialogues, the average number of turns for UMSR remained almost constant at 9.41, whereas TownInfo showed an increase reaching up to 24.19. This huge difference is obviously a significant improvement in system efficiency and user satisfaction. It also supports our hypothesis that the UMSR approach can present trade-offs understandably. For dialogues without a trade-off the number of turns was 9.07 for UMSR and 11.37 for TownInfo.

Dialogue duration also showed a great improvement in UMSR over TownInfo (4:49 (m:s) vs. $6: 11$ ). The duration however was almost the same for the two systems when a trade-off existed (4:40 vs. 4:49). This could mean that although the number of turns in this case is smaller for UMSR, the length of the generated output is longer, and requires more attention to understand. Yet again in dialogues without a trade-off, UMSR had a considerably shorter duration than TownInfo (4:57 vs. 7:34).

Average scores of the questionnaires are given in Table 2.
In response to the question "I thought the way the system provided information to me was easy to understand" the average score over all 108 dialogues was 5.04 for UMSR and 4.87 for TownInfo. The preference for UMSR exists for dialogues both with and without a trade-off. However, for all three cases the differences were not significant $(p>0.05)$.

Conciseness is the quality of providing a concise overview of all the available options to the user. The UMSR system was preferred at 4.65 over 4.04 for TownInfo $(p=0.034)$. The difference between the two systems is very significant for dialogues with a trade-off $(p<0.003)$. However, for dialogues without a trade-off $p=0.92$. This was predictable as the main innovation in UMSR is the ability to present trade-offs in a concise and understandable way, hence the significant difference for the dialogues with trade-offs.

To evaluate their confidence in having heard all the relevant options, the subjects were asked to rate the statement "I thought there were better options for my request than what the system gave me". Because of the negative nature of the question, the Likert scale was inverted before analysis. The average score was 3.22 and 2.93 for UMSR and TownInfo respectively. This indicates that the users have slightly more confidence in having heard all the relevant options with the UMSR system, although this difference is not significant $(p>0.05)$. For dialogues with a trade-off, the average confidence score was slightly better for TownInfo (2.74 vs. 2.67), but not significant ( $p=$ $0.8)$. However, there is a significant difference for dialogues without a trade-off $(p<0.03)$. Another notable issue is the overall low scores for the cases with a trade-off. This signifies that perhaps more information needs to be given to the user for dialogue turns describing a trade-off. A careful balance needs to be drawn between conciseness and comprehensiveness in these cases. This however, will obviously increase dialogue duration, and might affect understandability.

By accessibility, we mean ease of use and communication with the system. The scores for UMSR and TownInfo were 3.66 and 3.20 respectively $(p=0.18)$. A more significant difference in accessibility was noted for dialogues with a trade-off $(p=0.008)$. Again it seemed that users preferred UMSR when it came down to dealing with trade-offs. However, the accessibility scores for dialogues without a trade-off were almost the same $(p=0.92)$. 
Efficiency is the quality of enabling users to find the optimal option quickly. The statement "In this task, the system allowed me to find the optimal restaurant quickly", resulted in an average score of 4.69 for UMSR vs. 3.59 for TownInfo $(p=0.002)$. Once again, a significant difference was noted for dialogues with a trade-off, with 4.15 and 2.70 for UMSR and TownInfo respectively $(p=0.004)$. However, the difference for dialogues without a trade-off was not significant $(p=0.12)$.

\section{Conclusions and Future Work}

In this paper, we evaluated the effectiveness of the UMSR approach in information presentation in a full end-to-end dialogue system. The UMSR approach was compared with the traditional sequential enumeration of options. Our results verified our hypothesis that the UMSR approach presents a better overview of the trade-offs within the option space, and improves user experience and confidence in the system. Furthermore, with the UMSR approach there is a significant reduction in the number of dialogue turns required to complete the task. The results also showed that UMSR specifically outperforms TownInfo when there is a tradeoff involved. The UMSR results presented statistically significant improvement for conciseness, accessibility, and efficiency. Overall, subjects were more satisfied with the UMSR system. When they were asked if they would use the system again as a deployed product the score was 4.74 for UMSR and 3.70 for TownInfo $(p=0.002)$, further verifying that the users preferred the UMSR approach over the sequential enumeration of TownInfo.

In future work we intend to make a number of improvements. For example in the turn generation algorithm, we will optimise the generated output in an effort to strike a balance between understandability and complexity. Another important issue is to modify the UMSR algorithm so that it can accept multiple attributes in a single query. Moreover, we will perform experiments with both speech input and output. Finally, we will compare the UMSR approach with the UM and SR approaches in the same setting, i.e., a full end-to-end SDS.

\section{Acknowledgements}

This paper is based on a research experiment conducted at the University of Edinburgh. Paksima was funded by the European Commission Erasmus Mundus scholarship program. Georgila was partially funded by the Wellcome Trust VIP Award and is currently funded by the U.S. Army Research, Development, and Engineering Command (RDECOM). The content does not necessarily reflect the position or the policy of the U.S. Government, and no official endorsement should be inferred. The authors thank the three anonymous reviewers.

\section{References}

G. Carenini and J.D. Moore. 2001. An empirical study of the influence of user tailoring on evaluative argument effectiveness. In Proc. of IJCAI.

G. Chung. 2004. Developing a flexible spoken dialog system using simulation. In Proc. of ACL.

V. Demberg and J.D. Moore. 2006. Information presentation in spoken dialogue systems. In Proc. of EACL.

S. Elzer, J. Chu-Carroll, and S. Carberry. 1994. Recognizing and utilizing user preferences in collaborative consultation dialogues. In Proc. of UM '94.

J. Henderson, O. Lemon, and K. Georgila. 2008. Hybrid reinforcement/supervised learning of dialogue policies from fixed datasets. Computational Linguistics, 34(4):487-511.

O. Lemon, K. Georgila, J. Henderson, and M. Stuttle. 2006. An isu dialogue system exhibiting reinforcement learning of dialogue policies: generic slot-filling in the talk in-car system. In Proc. of EACL.

J.D. Moore, M.E. Foster, O. Lemon, and M. White. 2004. Generating tailored, comparative descriptions in spoken dialogue. In Proc. of FLAIRS.

J. Polifroni and M. Walker. 2008. Intensional summaries as cooperative responses in dialogue: $\mathrm{Au}-$ tomation and evaluation. In Proc. of $A C L-H L T$.

J. Polifroni, G. Chung, and M. Walker. 2003. Towards automatic generation of mixed-initiative dialogue systems from web content. In Proc. of Eurospeech.

M.A. Walker, R.J. Passonneau, and J.E. Boland. 2001. Quantitative and qualitative evaluation of darpa communicator spoken dialogue systems. In Proc. of $A C L$.

M. Walker, S. Whittaker, A. Stent, P. Maloor, J.D. Moore, M. Johnston, and G. Vasireddy. 2004. Generation and evaluation of user tailored responses in dialogue. Cognitive Science, 28:811-840.

A. Winterboer and J.D. Moore. 2007. Evaluating information presentation strategies for spoken recommendations. In RecSys '07: Proc. of the 2007 ACM conference on Recommender systems. 


\section{Appendix}

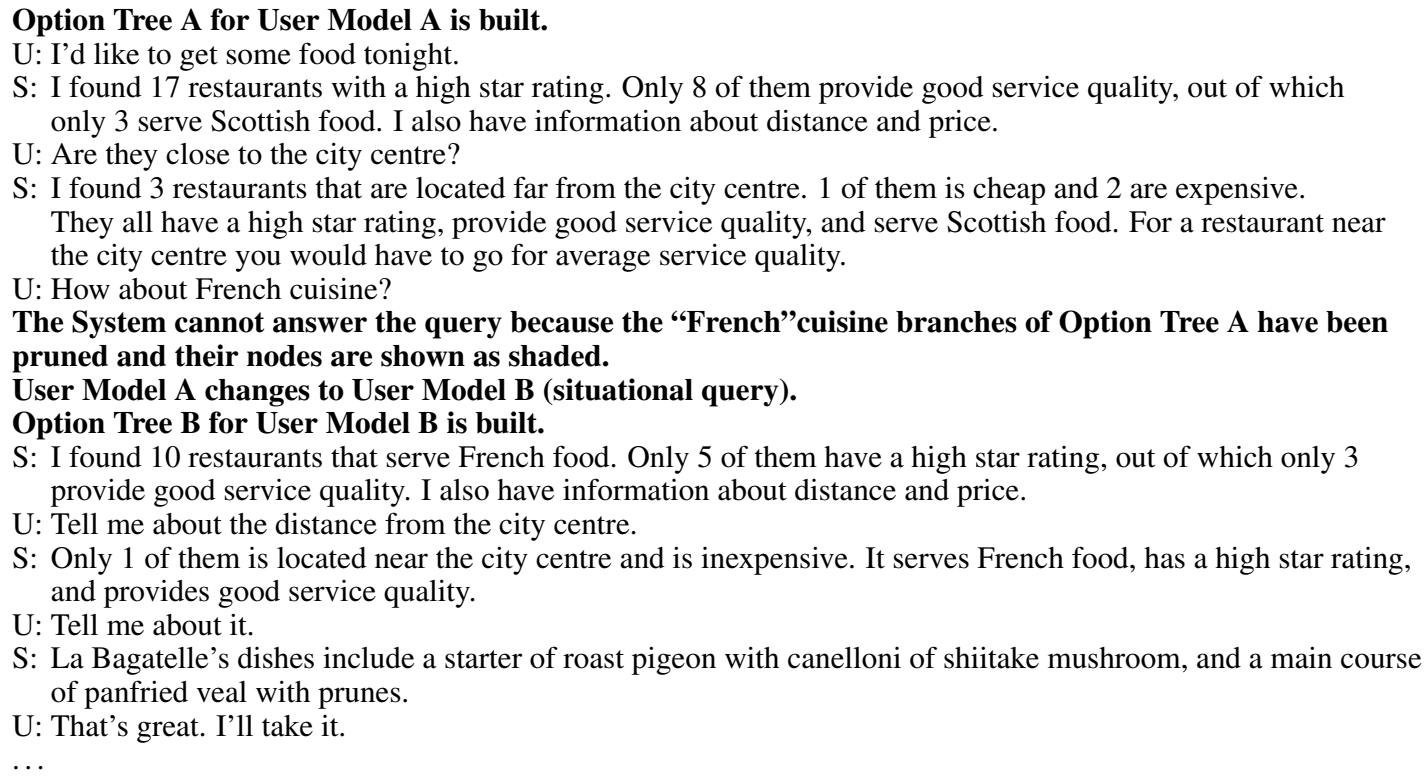

S: I found 10 restaurants that serve French food. Only 5 of them have a high star rating, out of which only 3 provide good service quality. I also have information about distance and price.

$\mathrm{U}:$ Tell me about the distance from the city centre.

$\mathrm{S}$ : Only 1 of them is located near the city centre and is inexpensive. It serves French food, has a high star rating, and provides good service quality.

$\mathrm{U}$ : Tell me about it.

S: La Bagatelle's dishes include a starter of roast pigeon with canelloni of shiitake mushroom, and a main course of panfried veal with prunes.

U: That's great. I'll take it. $\cdots$

Figure 6: Example dialogue with our UMSR approach for a business person, User Model A (Table 3) and User Model B (Table 4), Option Tree A (Fig. 7) and Option Tree B (Fig. 8).

\begin{tabular}{|cccc|}
\hline UserID & Attribute & Value & Rank \\
\hline 2 & Star & High & 1.00 \\
2 & Service & Good & 2.00 \\
2 & Cuisine & Scottish & 3.00 \\
2 & Distance & Near & 4.00 \\
2 & Price & Don't Care & 5.00 \\
\hline
\end{tabular}

Table 3: Sample user model for a business person (User Model A).

\begin{tabular}{|cccc|}
\hline UserID & Attribute & Value & Rank \\
\hline 2 & Cuisine & French & 0.95 \\
2 & Star & High & 1.00 \\
2 & Service & Good & 2.00 \\
2 & Distance & Near & 4.00 \\
2 & Price & Don't Care & 5.00 \\
\hline
\end{tabular}

Table 4: Modified user model for a business person after the situational query "I'd like a French restaurant" (User Model B). 


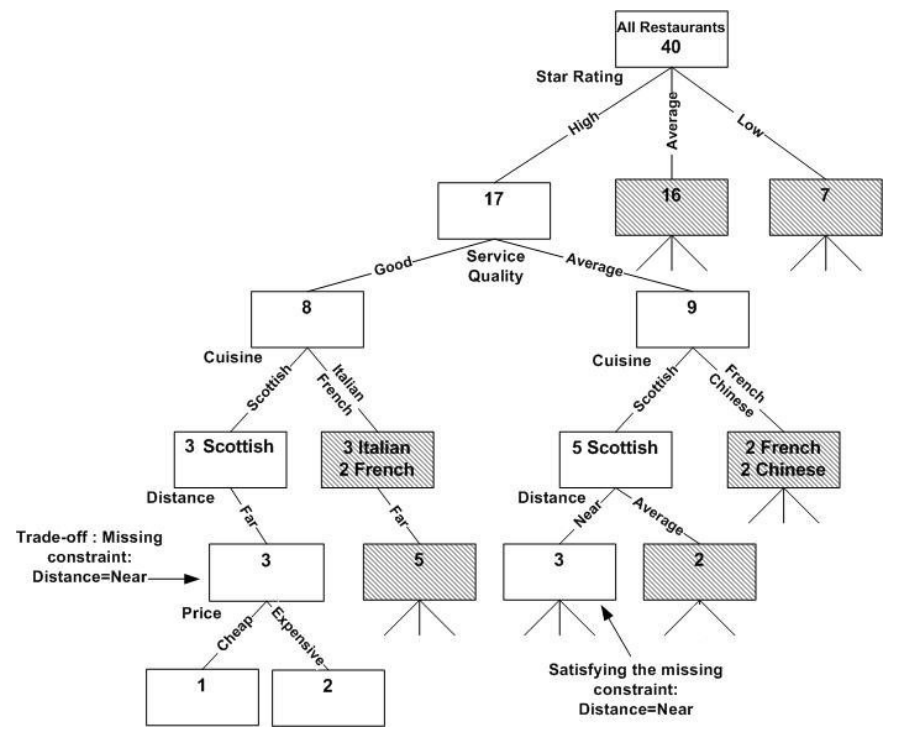

Figure 7: Option tree structure (Option Tree A) corresponding to the User Model A of Table 3. Pruned nodes are shown as shaded.

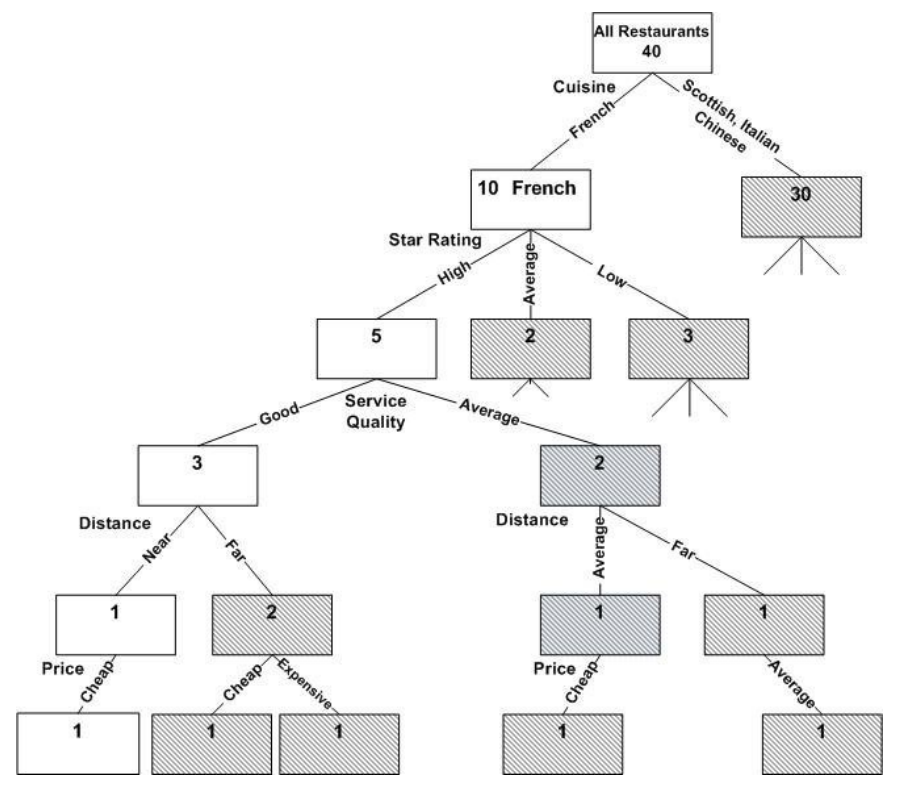

Figure 8: Option tree structure (Option Tree B) corresponding to the User Model B of Table 4. Pruned nodes are shown as shaded. 